- This paper gives an insight into primary healthcare professionals' perceptions and knowledge of oral cancer.

- The paper highlights potential training needs of medical and dental team members.

- It identifies potential methods for delivering oral cancer educational programmes.

\title{
The role of primary healthcare professionals in oral cancer prevention and detection
}

\author{
L. M. D. Macpherson, ${ }^{1}$ M. F. McCann, ${ }^{2}$ J. Gibson, ${ }^{3}$ V. I. Binnie ${ }^{4}$ and K. W. Stephen ${ }^{5}$
}

\begin{abstract}
Aim To investigate current knowledge, examination habits and preventive practices of primary healthcare professionals in Scotland, with respect to oral cancer, and to determine any relevant training needs. Setting Primary care.

Method Questionnaires were sent to a random sample of 357 general medical practitioners (GMPs) and 331 dental practitioners throughout Scotland. Additionally, focus group research and interviews were conducted amongst primary healthcare team members.

Results Whilst $58 \%$ of dental respondents reported examining regularly for signs of oral cancer, GMPs examined patients' mouths usually in response to a complaint of soreness. The majority of GMPs (85\%) and dentists (63\%) indicated that they felt less than confident in detecting oral cancer, with over $70 \%$ of GMPs identifying lack of training as an important barrier. Many practitioners were unclear concerning the relative importance of the presence of potentially malignant lesions in the oral cavity. A high proportion of the GMPs indicated that they should have a major role to play in oral cancer detection (66\%) but many felt strongly that this should be primarily the remit of the dental team. Conclusion The study revealed a need for continuing education programmes for primary care practitioners in oral cancer-related activities. This should aim to improve diagnostic skills and seek to increase practitioners' participation in preventive activities.
\end{abstract}

\section{INTRODUCTION}

Since the mid-1970s, the incidence of oral cancer has been rising steadily in most European countries, ${ }^{1,2,3}$ with rates in Scotland being higher than those for England and Wales. ${ }^{4}$ In the UK, oral cancer is estimated to account for $1-4 \%$ of all malignancy, ${ }^{5}$ with an incidence of approximately 3,500 cases per year. ${ }^{6}$

${ }^{1}$ Senior Lecturer and Honorary Consultant in Dental Public Health, ${ }^{2}$ General Dental Practitioner, Glasgow/ Part-time Lecturer, Primary Dental Care (now at St Andrews House, Regent Road, Edinburgh), ${ }^{3}$ Senior Lecturer and Honorary Consultant in Oral Medicine,

${ }^{4}$ Lecturer in Oral Health Promotion / Dental Public Health, ${ }^{5}$ Emeritus Professor of Dental

Public Health, University of Glasgow Dental School.

${ }^{*}$ Correspondence to: Lorna Macpherson, University of Glasgow Dental School, 378

Sauchiehall Street, Glasgow, G2 3JZ.

Email: I.macpherson@dental.gla.ac.uk

\section{Refereed paper}

Received 04.03.02; Accepted 29.01.03

doi:10.1038/sj.bdj.4810481

๑ B British Dental Journal 2003; 195: 277-281
In Scotland, between 1980 and 1995, increases of 100\% and 60\%, respectively, in the incidence of cancer of the 'mouth' and tongue, were noted, with the corresponding rise in deaths being 69\% and $64 \%$. The disease has historically been associated mainly with older age groups, but the increase has been particularly evident amongst younger adults, ${ }^{7}$ as highlighted in The Oral Health Strategy for Scotland. ${ }^{8}$ Here oral cancer was identified as a priority area for action.

Between the mid-1970s and 1990s, oral cancer 5-year survival rates have declined in Scotland, ${ }^{9}$ and from 1991-1995, for sites ICD9, 140-149, they were only $40 \%$ and $53 \%$ for males and females aged 15-74 years, respectively. ${ }^{10}$

These worsening survival rates may be related in part to a change in case mix, with an increase in proportion of patients from deprived areas, ${ }^{11}$ and also to the continuing problems of late presentation and diagnosis of the disease. ${ }^{12}$

Compared with most sites, the oral cavity is readily accessible to examination. Thus oral cancer should be amenable to early detection; and treatment at the premalignant or early malignant phase has been shown to be associated with improved survival rates. ${ }^{13,14}$ Hence, early detection must constitute an important element in reducing the high morbidity and mortality from oral cancer.

Several risk factors for oral cancer have been identified, notably the use of tobacco and alcohol, which are known to act synergistically. ${ }^{15,16}$ In common with many other diseases, incidence is higher among those from low socio-economic groups, with Scottish rates 3.5 times higher in DEPCAT 7 males compared with those in DEPCAT $1 .{ }^{10}$

In 1993, a UK working group on screening for oral cancer recommended that the procedure should be carried out opportunistically. ${ }^{17}$ However, while examination of the oral cavity for squamous cell carcinoma and other mucosal abnormalities should form part of a routine dental 'check-up', it is known that those in the 'high risk' groups (ie smokers and those from lower socio-economic groups) are least likely to visit a dentist on a regular basis. ${ }^{18}$ Additionally, among the over-50s, dental registration rates fall markedly with increasing age. Conversely, however, such groups are more likely to visit members of the primary care medical team. Thus, it has been proposed that, while efforts should be made to increase adult dental registration rates, other primary care health professionals also have important roles to play in attempting to improve early detection rates and consequent treatment outcomes associated with oral cancer. ${ }^{6}$ 
The aims of this investigation were to carry out quantitative and qualitative research among primary healthcare professionals in Scotland to:

1. Assess knowledge and awareness of oral cancer;

2. Assess current practice in relation to the detection of lesions and referral of patients;

3. Determine perceptions regarding their role in the detection and prevention of oral cancer; and

4. Assess training needs in relation to oral cancer.

While some results relating to the questionnaire survey of dentists have been reported previously, ${ }^{19}$ this paper compares responses of medical and dental practitioners and presents the findings of the qualitative aspect of the study.

\section{MATERIALS AND METHODS}

\section{Quantitative research}

Postal questionnaires were sent to a random sample of general medical and dental practitioners from across Scotland, similar questionnaires being used for both groups. Following evaluation of a pilot study, the questionnaire was distributed in 1998, along with an introductory letter, to 357 general medical practitioners (GMPs), representing a 1:10 sample. The dental practitioners surveyed included both those working within general dental practice and the community dental service. A random sample of 1:6 GDPs was drawn, providing a selection of 232, and a further 99 questionnaires were distributed, via community service managers, to community dental officers (CDOs), representing a 1:2 sample. The proportion of CDS practitioners was higher than general practitioners to enable representation of the former group from different geographical and socio-economic areas in Scotland, and to allow for the variation in the duties of individual members of the CDS.

For both medical and dental groups, a stratified sampling technique was used, after selection of the first recipient via a random numbers table. A follow-up mailing to non-respondents was carried out after an interval of three weeks. The questionnaire had 34 questions and was estimated to take between 15 and 20 minutes to complete, the five main themes having been described previously. ${ }^{19}$

Analyses of the data were carried out using the SPSS for Windows software package. Frequency tables were produced and cross-tabulations performed where appropriate.

\section{Qualitative research}

The qualitative phase of the investigation was conducted by an independent market research organisation at around the same time period as the questionnaire study. The study focused on areas less amenable to quantification. The key issues discussed with the health professionals were: an identification and elaboration of factors influencing perceptions, awareness and understanding of oral cancer; an exploration of factors influencing the motivation of health professionals to engage in opportunistic screening; and an assessment of the professionals' views on training needs and the type of material which should be developed to inform health professionals about oral cancer.

Individual, semi-structured interviews were carried out with general medical and dental practitioners, community dental officers, dental hygienists and community pharmacists. Interview schedules were developed in consultation with the authors. Respondents were encouraged to answer in their own words, and to expand on answers where they felt this to be appropriate. Focus groups were used to explore the views of health visitors, practice and district nurses.

The qualitative strand was conducted in Lothian, Greater Glasgow, Tayside and Grampian Health Board areas, to ensure a Scotland-wide perspective. Lists of relevant health professionals were obtained from primary care trusts and individuals were selected randomly from areas where levels of deprivation were known to be higher (as defined by the level of deprivation associated with the practice/pharmacy postcode).

A total of 11 interviews was completed with general medical practitioners, ten with dental practitioners, six with community dental officers, three with dental hygienists and three with community pharmacists. Three focus groups were run with health visitors, practice and district nurses in Glasgow, Lothian and Tayside.

\section{RESULTS}

\section{Response rate}

For the quantitative phase of the research, completed questionnaires were returned by 198 medical practitioners, representing a response rate of 57\%. Eight uncompleted forms were returned by practices where the intended recipient was no longer present. Dental practitioners completed a total of 225 valid questionnaires, a response rate of $68 \%$. Overall, a total of 686 questionnaires was distributed, resulting in 423 valid returns, and an overall response rate of $62 \%$.

Just over half (56\%) of GMP respondents were male and 44\% female. A third of medical practitioners returning completed questionnaires had been qualified for more than 20 years, 17\% for between $16-20$ years, with 20\% having graduated in the previous 10 years. For the dental practitioner strand, 59\% were male, and $41 \%$ female. Of these, a third of respondents (33\%) had been graduated for more than 20 years, 16\% between 16-20 years, $18 \%$ from 6-10 years, with 14\% graduated for less than five years.

Comparison of socio-demographic characteristics of responders and non-responders within the dental strand of the study have been reported previously. ${ }^{19}$ A slightly higher proportion (18\% v $13 \%$ ) of the practices of non-respondents were located in areas of socio-economic deprivation, with more being located in inner city areas (27\% v 18\%).

\section{Risk factors}

Almost all medical practitioners (97\%) identified smoking as an important risk factor for oral cancer, with alcohol consumption (79\%) and age (76\%) also perceived to be important. Comments received from GMPs in the qualitative strand of the study, indicated a high awareness of smoking as a cause of oral cancer but less so for the role of alcohol in the disease: 'Trauma, probably smoking, denture wear causing ulceration... I don't know about the alcohol factor, although I see no reason why it shouldn't be a factor as it affects your health in lots of other ways.' Similarly, the vast majority of dentists surveyed recognised the importance of these, with $94 \%$ of respondents identifying smoking as a risk factor and 90\% being aware of the importance of alcohol consumption.

Trauma was considered an important risk factor by $43 \%$ of GMPs, whereas only 32\% of dentists felt similarly. Fungal and viral infections were deemed to be of greater importance in the aetiology of oral cancer by dental respondents, with 33\% perceiving fungal infections to be relevant as compared with $20 \%$ of medical practitioners. Of the dental practitioners, 35\% considered viruses to be important, with only $23 \%$ of medical colleagues believing this to be so.

The respondents' perception of the relative status of various oral conditions as predisposing factors relating to the aetiology of oral cancer is shown in Table 1 . Whilst a high proportion of GMPs (72\%) and GDPs (79\%) considered leukoplakia a 'very important' potentially malignant lesion, a lower percentage of practitioners rated erythroplakia in the same manner $(22 \%$ and $66 \%$ respectively). As will be seen in the discussion section, this is a misconception which requires to be addressed. 
Table 1 Respondents perceptions of relative importance of oral conditions in the aetiology of oral cancer

\begin{tabular}{|c|c|c|c|c|c|c|c|c|}
\hline \multirow{3}{*}{ Oral Condition } & \multicolumn{8}{|c|}{ Perceived importance } \\
\hline & \multicolumn{2}{|c|}{ Very important $\mathrm{N}(\%)$} & \multicolumn{2}{|c|}{ Important N (\%) } & \multicolumn{2}{|c|}{ Unimportant N (\%) } & \multicolumn{2}{|c|}{ Don't know N (\%) } \\
\hline & GMP & GDP & GMP & GDP & GMP & GDP & GMP & GDP \\
\hline Aphthae & 7 (4) & $10(4)$ & $35(18)$ & $42(19)$ & $127(64)$ & $156(69)$ & $29(15)$ & $17(8)$ \\
\hline Chronic candidosis & 13 (7) & $61(27)$ & $84(42)$ & $124(55)$ & $57(29)$ & $32(14)$ & $44(22)$ & $8(4)$ \\
\hline Erythroplakia & $43(22)$ & $150(67)$ & $49(25)$ & $62(28)$ & $9(4)$ & $4(2)$ & $97(49)$ & $9(4)$ \\
\hline Leukoplakia & $143(72)$ & $178(79)$ & $43(22)$ & $39(17)$ & 1 (1) & $4(2)$ & 11 (6) & $4(2)$ \\
\hline
\end{tabular}

\begin{tabular}{|c|c|c|}
\hline & $\begin{array}{l}\text { Medical practitioners }(\mathrm{N}=198) \\
\text { frequency }(\%)\end{array}$ & $\begin{array}{l}\text { Dental practitioners }(\mathrm{N}=225) \\
\text { frequency }(\%)\end{array}$ \\
\hline Very confident & $5(2)$ & $12(5)$ \\
\hline Confident & $25(13)$ & $73(32)$ \\
\hline Moderately confident & $93(47)$ & $99(44)$ \\
\hline Not confident & $69(35)$ & $39(17)$ \\
\hline No response & $6(3)$ & $2(1)$ \\
\hline
\end{tabular}

\section{Examination practices}

The medical practitioners indicated that they examined patients' mouths usually in response to a complaint of soreness (94\%), or as a result of the practitioner's knowledge of a pre-existing oral condition $(81 \%)$.

Of the dental respondents, 58\% indicated they always carried out an examination for oral cancer during the course of the routine dental examination, with a further $38 \%$ doing so on an occasional basis.

\section{Perceptions and awareness of oral cancer}

The qualitative phase of the study sought to investigate respondents' knowledge of the main symptoms and signs of oral cancer. Most of those questioned demonstrated some awareness of the common signs of the disease.

When asked to indicate what they felt might constitute an abnormal mouth, respondents mentioned red or white patches, but indicated they would be alert to any condition appearing to deviate from normality which they were unable to categorise. Practice nurses acknowledged a key range of signs and symptoms when questioned about the clinical presentation of the disease:

'A sore that won't heal.' 'Mouth ulcers, swellings.' 'Difficulty swallowing.'

However, there was also some uncertainty:

'Possibly problems with taste - losing your sense of taste'

'Dental problems with the teeth...decaying teeth and pain with chewing things on the teeth, losing a lot of teeth'.

Community pharmacists mentioned 'non-healing ulcers' and 'growths' as possible problems. However, one community pharmacist highlighted the fact that public understanding of the potential seriousness of oral problems was limited, with patients tending to view oral mucosal abnormalities, such as white patches, as unimportant. Some members of the public dismissed such findings as unrelated to either dental or general health:

'I think it's because they don't perceive it as being something serious. You know if you get a pain in your side and it comes and goes when you eat, you think "I'll need to get that checked out". Whereas, with your mouth, you can open that and have a look in the mirror and say "Oh aye, that wee thing" '.

\section{Practitioner confidence}

Only 15\% of medical practitioners considered themselves to be at least 'confident' of detecting pre-malignant or malignant oral lesions, in contrast to dental colleagues, of whom 37\% admitted to this level of confidence (Table 2).

Only 23\% of medical practitioners surveyed expressed confidence in their ability to assess whether an observed lesion merited urgent referral. A higher proportion of general dental practitioners and community dental officers were assured regarding assessing the need for an urgent referral, with some $48 \%$ of those responding to the survey indicating they were, at least, "confident'.

\section{Referral practices}

Over half of medical respondents (57\%) stated they would consider urgent referral if an intra-oral lesion had been present for four to five weeks, with $37 \%$ expressing the need for referral after two to three weeks. The vast majority of GMPs indicated that they would refer to general hospitals (74\%) while a further $22 \%$ said they would use a dental hospital facility. Of those medical practitioners who indicated that they would refer to general hospitals, 83\% said they would choose an oral and maxillofacial surgery unit, with a further 15\% opting for an ENT opinion. Only 1\% questioned would refer patients to a general surgery unit.

The majority of dental respondents (54\%) revealed they would refer suspicious lesions after two to three weeks, with a further $30 \%$ doing so after four to five weeks' observation. While the majority of dental respondents indicated they normally referred to a dental hospital (56\%), a substantial number contacted general hospitals (43\%). Of the latter group, the department most commonly selected was the oral and maxillofacial surgery unit.

\section{Perceived barriers to oral cancer examination}

It was felt that financial constraints were likely to deter some atrisk individuals from visiting the dentist, and that the general medical practitioner would be in a particularly good position to 'opportunistically screen' such individuals.

'The doctor is the first person a lot of these people (elderly, edentulous) tend to go to because he's free. If you go to the dentist, it's going to cost you money if you're not exempt.'

However, overall, medical practitioners felt that the dentist was the mouth specialist.

'It's all down to the training of doctors and dentists, because dentists are the ones that know the mouth. They tend to know the mouth a lot better than the doctors because they're seeing mouths every day. Doctors are looking at the whole body.'

Community nurses indicated they were often in a position to screen opportunistically for oral cancer, but were not doing so at present. This group usually found itself dealing with patients' immediate presenting problems during the course of a consultation.

Almost all the medical practitioners who replied to the survey felt they had some role to play in oral cancer detection, with $66 \%$ stating this role to be 'major'. However, over 70\% identified lack of training as an important barrier to undertaking an oral cancer examination, and more than one third (37\%) revealed they had never received any organised tuition on the subject. Medical practitioners also said they perceived time to be an important barrier to undertaking an oral cancer examination, with approximately half (47\%) identifying 'lack of time' as a significant barrier. 
One GMP expressed the following view:

'I think it's a time issue. Ideally, we'd like to do it, but we don't have the time or the resources.'

Others felt very strongly that opportunistic screening was definitely the province of the dentist:

'I think it should be a dentist. I don't think it should even be entertained to do it in general practice. It should be a dental screening.'

Amongst dental respondents, $41 \%$ claimed lack of training to be an important barrier to undertaking an examination for oral cancer. However, 31\% confirmed that the potential to generate patient anxiety constituted a barrier, this having been identified during discussions with GDPs at the pilot stage of the study as a perceived barrier to oral cancer screening. Other deterrents identified by GDPs were time (43\%) and the NHS remuneration system (40\%).

\section{Health promotion and prevention of oral cancer}

A high proportion (87\%) of GMPs indicated they routinely made enquiries of their patients in relation to smoking habits, and 67\% asked routinely about alcohol consumption. With respect to oral cancer prevention, some respondents in the qualitative strand of the investigation indicated a certain fatalistic view of the impact of preventive initiatives on the progress of the disease, with one medical practitioner commenting:

'I don't know how it could be prevented. But, there again, if we're saying it's to do with smoking and alcohol, then it could certainly be reduced.'

Some dental respondents were equally sceptical:

'I don't think it can be prevented. I don't think any cancer can be prevented........'

The proportion of dental respondents reporting receiving past training in counselling on smoking or alcohol, were $3 \%$ and $1 \%$, respectively. Only 19\% of dental respondents routinely made enquiries into smoking habits, with a further $49 \%$ doing so 'occasionally'.

Only seven dental respondents (3\%) routinely asked patients about alcohol use, with the majority (68\%) rarely or never doing so. Their answers indicated they felt uncomfortable making enquiries about alcohol use:

'embarrassing'; 'bad PR; not my business'; 'Patients get very offended'; and 'enquire only if patient under the influence'.

Whilst relatively few medical practitioners had undergone formal training in relation to counselling regarding smoking (26\%) and alcohol usage (32\%), most expressed confidence in giving advice to patients on these topics ( $70 \%$ and $64 \%$, respectively), and $90 \%$ of GMPs indicated this was already being carried out by other members of the primary care medical team.

Most medical practitioners identified general dental practitioners (91\%) and dental hygienists (79\%) as having major roles to play in oral cancer prevention, while 56\% felt GMPs should also have a prominent input. Approximately one quarter of the GMPs identified an important function for other members of the primary care team, notably practice nurses and health visitors (Table 3).

\section{Training needs}

The vast majority of medical practitioners wished for more training in both the detection (91\%) and prevention (79\%) of oral can- cer, with 67\% of GMPs indicating they wanted additional advice on referral pathways. The percentage of dentists desiring further oral cancer detection and prevention training were $92 \%$ and $78 \%$, respectively. Dental respondents also indicated a wish for further training with respect to advice on smoking (60\%) and alcohol (61\%), particularly in relation to information on groups to which patients could be referred for counselling.

Responses to the qualitative component of the study indicated that preferences for the format of training were not professionspecific. Attendance at courses were thought by many to provide an opportunity to interact with colleagues, and to enjoy the benefits of expert instruction and good visual material. However, due to constraints on time and geography this was not always an option for all, and many participants requested access to an appropriate distance learning package. Concise written material which could be held within the practice and good quality photographs and other visual aids were felt to be essential. For those with access to computers, many thought a CD-Rom would be useful.

\section{DISCUSSION}

At present, only 50\% of adults are registered with an NHS general dental practitioner in Scotland. ${ }^{20}$ Even allowing for patients attending on an occasional basis, and those receiving care privately, there are clearly many Scots adults who do not regularly access dental primary care services. Thus it is recognised that opportunistic screening for oral cancer, if focused only on the dental examination, would fail to reach large proportions of the at-risk population.

Comments received in the qualitative strand of this study indicate that both medical and dental professionals accept that many at-risk groups do not attend the dentist regularly, and that such individuals were more likely to have contact with the medical practice, indicating a clear role for the primary care medical team.

However, the present study also reveals the need for wider involvement of primary care professionals, beyond that of medical and dental personnel. Community pharmacists highlighted the fact that, for many individuals, the accessibility of the oral tissues means that lesions in this part of the body are often the subject of self-diagnosis and medication. Thus, the community pharmacist may in fact, be the first (or only) point of professional contact for many members of the public, and therefore may have a vital role to play in directing those with early lesions to primary care medical or dental practitioners. In recent years, this issue has been highlighted in Scottish pharmaceutical postgraduate education. ${ }^{21}$

The study achieved an overall response of $62 \%$, with the dental strand returning $11 \%$ more than the medical practitioners, perhaps reflecting how these distinct groups perceived their respective roles in the prevention and detection of oral cancer. While the medical response, in particular, was disappointing, it is possible that those who believed the study to be professionally relevant may have been more likely to respond, thus introducing the potential for bias, which must be borne in mind when interpreting these findings. Nonetheless, the need for the medical practitioner to be aware of both the risk factors and early signs of oral cancer was highlighted by Dawson et al. ${ }^{12}$ substantial numbers of oral cancer referrals having been recorded from this source. ${ }^{13}$

\begin{tabular}{|c|c|c|c|c|c|c|c|c|}
\hline & \multicolumn{2}{|c|}{ Major N (\%) } & \multicolumn{2}{|c|}{ Minor N (\%) } & \multicolumn{2}{|c|}{ None N (\%) } & \multicolumn{2}{|c|}{ No response $\mathrm{N}(\%)$} \\
\hline & GMP & GDP & GMP & GDP & GMP & GDP & GMP & GDP \\
\hline Community pharmacist & $20(10)$ & $20(9)$ & $96(48)$ & $133(59)$ & $56(28)$ & $57(25)$ & $26(13)$ & $15(7)$ \\
\hline Dental hygienists & $156(79)$ & $141(63)$ & 31 (16) & $76(34)$ & $2(1)$ & $0(0)$ & $9(4)$ & $8(4)$ \\
\hline Dentists & $181(91)$ & $203(90)$ & $11(6)$ & $14(6)$ & $0(0)$ & $0(0)$ & 6 (3) & $8(4)$ \\
\hline Medical practitioners & $111(56)$ & $181(80)$ & $75(38)$ & $33(15)$ & $1(1)$ & $0(0)$ & $11(6)$ & $11(5)$ \\
\hline Health promotion officers & $90(45)$ & $136(60)$ & $72(36)$ & $72(32)$ & 15 (8) & $9(4)$ & $21(11)$ & $8(4)$ \\
\hline Health visitors & $42(21)$ & $65(29)$ & $116(59)$ & $136(60)$ & $26(13)$ & $15(7)$ & $14(7)$ & $9(4)$ \\
\hline Practice nurses & $42(21)$ & $57(25)$ & $119(60)$ & $133(59)$ & $19(10)$ & $25(11)$ & $14(7)$ & $10(4)$ \\
\hline
\end{tabular}


There was a wide range of views expressed in the study with respect to the appropriate period of suspicious oral lesion observation, the recommended time being three to four weeks. ${ }^{6}$ While dental respondents tended to adhere to these guidelines more so than their medical colleagues, a substantial number of both respondent groups indicated they would delay referral until four to five weeks' observation and 6\% of dental, and 16\% of medical practitioners said they would wait even longer. Such a philosophy has implications for treatment outcomes, with prompt referral and early treatment improving chances of survival. ${ }^{13,14}$

While most practitioners (both GMP and GDP) correctly identified higher incidence rates of oral cancer amongst the elderly, over the past few decades a significant increase in cases has occurred among young adults. Thus, it must be appreciated that the disease can occur in either gender, at any age. Nonetheless, edentulous patients, who often see little need to attend a GDP after full denture provision, still require monitoring, as there was a general feeling amongst practitioners involved in oral cancer care, that chronic irritation and trauma may play a part in the causation and / or promulgation of the disease. Their views are supported by the work of Lockhart et al. ${ }^{22}$ where analysis of data from 28 intra-oral maligancies found all arose in areas contacting teeth and / or appliances.

Although the majority of medical practitioners stated they felt they had a major role to play in oral cancer prevention and detection, there was a general feeling that the dentist was the key primary care professional to undertake opportunistic oral cancer screening, with some GMPs expressing the view that this was beyond their remit. The present study data bear out these facts with results indicating that currently, many medical practitioners are not examining at-risk groups opportunistically, but only in response to a specific patient complaint, most commonly that of oral pain. This is an important finding, with training implications, as many precancerous and early malignant oral lesions are, in fact, painless. Consequently, if an intra-oral examination is carried out only in response to a complaint of pain, many early lesions may be missed, reducing the opportunity for early treatment. It may be that this is an indication of lack of time and the consequent need to prioritise, with oral cancer screening in medical practice perhaps targeted on those individuals at highest risk of contracting the disease.

Training should also help stress the benefits of early detection of oral cancer and pre-cancer, with their multiplicity of clinical presentations, especially including red patches, white patches, speckled patches and overt ulceration; areas where many practitioners expressed lack of confidence. Such recognition is of particular relevance, as literature reviews ${ }^{23,24}$ have reported that erythro-leukoplakia had a higher rate of malignant transformation $(9 \%-40 \%)$ than rates for all leukoplakias, where transformations of only $2 \%-6 \%$ were observed. These figures underpin the need for all practitioners to appreciate the imperative of identifying changes in the oral mucosa early, and to embark upon an appropriate management strategy.

The importance of smoking as a major risk factor for oral cancer in the UK should be emphasised in training, together with that of alcohol misuse. ${ }^{6}$ Unlike general dental practitioners, where only a very small proportion are currently involved in giving advice with respect to smoking cessation and sensible use of alcohol, the results confirmed that most medical practitioners do feel comfortable with this, and do offer advice to patients about such lifestyle factors. Thus this latter group, with high numbers of patient contacts, has the potential to reach large numbers of smokers, ${ }^{25}$ and is already contributing to oral health promotion through a holistic and common risk factor approach. In spite of the fact that GDPs may not have felt that alcohol-related questioning might be a good 'practice-builder', there is again a training-need requirement here.

The present study indicated that medical and dental practitioners are referring suspected oral cancer cases to a variety of centres.
This may be a reflection of geographic factors. However, ideally, patients should be seen in dedicated units which have the necessary expertise to co-ordinate treatment regimens and improve outcomes. Consideration is being given to this issue in development of oral cancer services through managed clinical networks.

In conclusion, while many GMPs perceive members of the dental team to have the greater role in the prevention and detection of oral cancer, they may be prepared to become more involved in examining the mouth, particularly those of high-risk patients. It could be that the key role for other members of the primary care medical team, notably practice and district nurses together with community pharmacists, is to identify at-risk patients and to facilitate access to an oral examination by either a dental surgeon or the patient's medical practitioner.

The current investigation reveals that further training is required at both undergraduate and postgraduate levels to increase awareness of oral cancer and its associated risk factors, and to strengthen primary care practitioners' abilities to diagnose potentially cancerous intra-oral lesions. To this end, a paper-, video- and websitebased distance learning package ${ }^{6}$ was developed recently and distributed to primary health care professionals in Scotland.

The authors are grateful to the primary health care professionals for their participation in the study and to Scottish Health Feedback for conducting the qualitative phase of the study. This investigation was funded by a grant from the Scottish Council for Postgraduate Medical and Dental Education.

1. Moller H. Changing incidence of cancer of the tongue, oral cavity and pharynx in Denmark. J Oral Pathol Med 1989; 18: 224-229.

2. Hindle I, Nally T. Oral cancer: a comparative study between England and Wales. Br DentJ 1991; 170: 15-20.

3. Cowan C G, Gregg TA, Kee F. Trends in the incidence of histologically diagnosed intra-oral squamous cell carcinoma in Northern Ireland, 1975-89. Br Dent J 1992; 173: 231-233.

4. Census, population and health group. Office for National Statistics. London: HMSO, 1999

5. Johnson N W, Warnakulasuriya K A A S. Epidemiology and aetiology of oral cancer in the United Kingdom. Community Dent Health 1993; 10: Supplement 1; 13-29.

6. Macpherson L M D, Gibson J, Binnie VI, Stephen K W. Oral cancer prevention and detection for the primary health care team. Glasgow: University of Glasgow, 2000.

7. Macfarlane G J, Boyle P, Scully C. Oral cancer in Scotland: changing incidence and mortality. Br Med J 1992; 305: 1121-1123.

8. Scottish Office Department of Health. Scotland's health: a challenge to us all. The oral health strategy for Scotland. Scottish Office. Edinburgh: HMSO, 1995.

9. Soutar D, Robertson G. Head and neck cancers. In Black R, Stockton D. Cancer scenarios: an aid to planning cancer services in Scotland in the next decade. Edinburgh: Scottish Executive, 2001.

10. Common Services Agency. Scottish Cancer Registry. Edinburgh: Scottish Cancer Intelligence Unit, Information and Statistics Division, 1999.

11. Macfarlane G J, Sharp L, Porter S. Trends in survival from cancers of the oral cavity and pharynx in Scotland: a clue as to why the disease is becoming more common. $\mathrm{Br} J$ Cancer 1996; 73: 805-808.

12. Dawson CE, Bain M, McCall D, Macpherson L M D, Moos K F. Scottish Needs Assessment Programme:oral cancer, Scottish forum for public health medicine, Glasgow, 1996.

13. Scully C, Malamos D, Levers B G H, Porter SR, Prime SS. Sources and patterns of referrals of oral cancer. BrMed J 1986; 293: 599-601.

14. Schnetler J F. Oral cancer diagnosis and delays in referral. Br J Oral Maxillofac Surg 1992; 30: 210-213.

15. Mashberg A, Boffetta P, Winkelman R, Garfinkel L. Tobacco smoking, alcohol drinking and cancer of the oral cavity and oropharynx among US veterans. Cancer 1993; 72: 1369-1375.

16. Llewelyn J, Mitchell R. Smoking, alcohol and oral cancer in South East Scotland: a ten year experience. Br J Oral Maxillofac Surg 1994; 32: 146-152.

17. Speight PM, Downer M C, Zakrewska J (Eds). Screening for oral cancer: should ora screening be introduced? Community Dent Health 1993; 10: Supplement 1, 1-3.

18. Haughney M G J, Devennie J C, Macpherson L M D, Mason D K. Integration of primary care dental and medical services: a three year study. Br DentJ 1998; 184:343-347.

19. McCann M F, Macpherson L M D, Binnie V I, Stephen K W. A survey of Scottish primary care dental practitioners' oral cancer-related practices and training requirements. Community Dent Health 2000; 17: 24-30.

20. NHS Scotland. Scottish Dental Practice Board Annual Report 2000/01. Scottish Dental Practice Board, Edinburgh, 2001.

21. Scottish Centre for Post Qualification Pharmaceutical Education. Scotland's Health, The challenge for pharmacy. University of Strathclyde, Glasgow, 1996.

22. Lockhart P B, Norris Jr C M, Pulliam C. Dental factors in the genesis of squamous cell carcinoma of the oral cavity. Oral Oncol 1998; 34: 133-139.

23. Scully C, El Kabir M. Candida and oral candidosis: a review. Crit Rev Oral Biol Med 1994; 5: 125-157.

24. Speight P M, Morgan PR. The natural history and pathology of oral cancer and precancer Community Dent Health 1993; 10 (Supplement 1): 31-41.

25. Secker-Walker RH et al. Comparisons of the smoking cessation counselling activities of six types of health professionals. Preventive Med 1994; 23: 800-808. 